Article

\title{
Synthesis of Novel 1,4-Naphthoquinones Possessing Indole Scaffolds Using In(OTf) 3 in Solvent-Free Conditions
}

\author{
Xiaojuan Yang ${ }^{1}$ and Liqiang $\mathrm{Wu}^{2, *}$ \\ 1 College of Chemistry and Chemical Engineering, Xinxiang University, Xinxiang 453003, Henan, China; \\ yangxiaojuan2005@126.com \\ 2 School of Pharmacy, Xinxiang Medical University, Xinxiang 453003, Henan, China \\ * Correspondence: 041110@xxmu.edu.cn; Tel.: +86-373-302-9879
}

Received: 11 July 2018; Accepted: 31 July 2018; Published: 6 August 2018

Abstract: Novel 1,4-naphthoquinones possessing indole scaffolds were prepared by the reaction of 2-hydroxy-1,4-naphthoquinone-substituted salicylic aldehydes and indoles using $\operatorname{In}(\mathrm{OTf})_{3}$ as a catalyst. The method has the advantages of simple operation, mild reaction conditions, and friendly environment.

Keywords: 1,4-naphthoquinone; substituted salicylic aldehydes; indoles; solvent-free; green synthesis

\section{Introduction}

Multi-component reactions, in which multiple reactants are combined to form a single, more complex system, have been used extensively to synthesize chemically and biologically important compounds [1]. This reaction provides a wide range of possibilities to efficiently construct highly complex molecules in one process, thus avoiding complex purification operations and saving solvents and reagents. In the past decade, the three component and four component reactions have undergone tremendous development [2].

Quinone skeletons are present in a broad range of natural products and synthetic molecules with important biological activities [3]. Among them, 1,4-naphthoquinone scaffolds have received considerable attention because of the synthetic challenges associated with their changeable molecular architecture and their interesting biological properties, such as their anticancer [4], antifungal [5], antiviral [6], and anti-inflammatory activities [7]. 1,4-Naphthoquinone skeleton can be found in a wide range of natural products, such as $\alpha$-lapachone [8], rhinacanthin C [9], and avicequinone C [10] (Figure 1). In this context, the development of facile methods to access these new targets with structural diversity is very desirable and valuable for drug discovery.<smiles>CC1(C)CCC2=C(O1)C(=O)c1ccccc1C2=O</smiles>

a-lapachone<smiles>CC(C)(O)c1cc2c(o1)C(=O)c1cccc(O)c1C2=O</smiles>

avicequinone $\mathrm{C}$<smiles>C/C=C(\C)CC/C=C(\C)C(=O)OCC(C)(C)CC1=C(O)C(=O)c2ccccc2C1=O</smiles>

rhinacanthin C

Figure 1. Structure of some natural 1,4-naphthoquinones.

Bioactive indole compounds are widely distributed in nature [11] and presented in the market [12] as active pharmaceutical ingredients $[13,14]$. Moreover, in modern times, indole derivatives are 
significant players in a diverse array of markets such as dyes, plastics, agriculture, vitamin supplements, over-the-counter drugs, flavor enhancers, and perfumery.

Herein, we describe a simple and efficient method for rapid preparation of 1,4-naphthoquinones possessing indole scaffolds using a catalytic amount of $\operatorname{In}(\mathrm{OTf})_{3}$ under solvent-free conditions (Scheme 1). As far as we know, it is probably the first example of synthesis 1,4-naphthoquinones possessing indole scaffolds using $\operatorname{In}(\mathrm{OTf})_{3}$ as catalyst under solvent-free conditions. As a result, 1,4-naphthoquinones-fused indoles, which combine two kinds of bioactive heterocyclic nuclei, are expected to become a research hotspot in pharmacology. Further studies to delineate the activities of the novel compounds are underway.

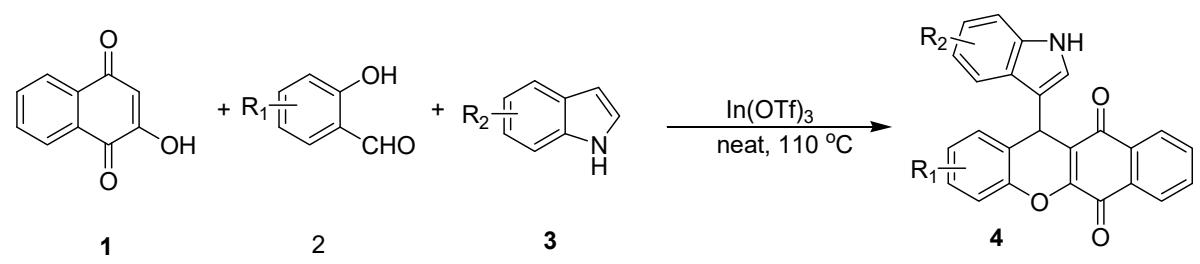

Scheme 1. Synthesis of 1,4-naphthoquinones possessing indole scaffolds.

\section{Results and Discussion}

Initially, we began our studies by evaluating the reaction between 2-hydroxy-1,4-naphthoquinone, salicylic aldehyde, and 2-phenylindole using $\operatorname{In}(\mathrm{OTf})_{3}(20 \mathrm{~mol} \%)$ as the catalyst in toluene as the solvent at $110{ }^{\circ} \mathrm{C}$ for $8 \mathrm{~h}$, which provided the desired product $4 \mathrm{a}$ in a $35 \%$ yield. Encouraged by this preliminary result, the screening of solvents and catalysts, as well as the in fluence of temperature and catalyst loading, was investigated to establish the optimized reaction conditions (Table 1). Solvent effects were first investigated (Table 1, compare entries 1-6). The neat reaction provided higher yields than those using other organic solvents (entries 2-6). To improve the yields, we then examined this reaction using different catalysts; the $\operatorname{In}(\mathrm{OTf})_{3}$ catalyst showed the best performance (entry 6). When this reaction was carried out without $\mathrm{In}(\mathrm{OTf})_{3}$ or in the presence of $p-\mathrm{TsOH}, \mathrm{H}_{2} \mathrm{SO}_{4}, \mathrm{FeCl}_{3}, \mathrm{Sc}(\mathrm{OTf})_{3}$, or $\mathrm{InCl}_{3}$, the product was obtained in low yield (Table 1, entries 7-12). The effect of the reaction temperature was investigated; it was observed that the reaction performed at $110{ }^{\circ} \mathrm{C}$ provided the best results (entries 6, 13-15). We also evaluated the amount of $\operatorname{In}(\mathrm{OTf})_{3}$ required for the reaction; the results from Table 1 (entries $6,16-18$ ) show that $10 \mathrm{~mol} \% \operatorname{In}(\mathrm{OTf})_{3}$ at $110{ }^{\circ} \mathrm{C}$ under solvent-free conditions is optimal for the reaction.

Table 1. Reaction conditions optimization for the synthesis $\mathbf{4 a .}$

\begin{tabular}{|c|c|c|c|c|c|}
\hline Entry & Solvent & Catalyst & Temperature $/{ }^{\circ} \mathrm{C}$ & Time/h & Yield $/ \%^{1}$ \\
\hline 1 & Toluene & $\operatorname{In}(\mathrm{OTf})_{3}(10 \mathrm{~mol} \%)$ & 110 & 8 & 35 \\
\hline 2 & DMF & $\operatorname{In}(\mathrm{OTf})_{3}(10 \mathrm{~mol} \%)$ & 110 & 8 & 39 \\
\hline 3 & $\mathrm{EtOH}$ & $\mathrm{In}(\mathrm{OTf})_{3}(10 \mathrm{~mol} \%)$ & reflux & 12 & 8 \\
\hline 4 & $\mathrm{CH}_{3} \mathrm{CN}$ & $\mathrm{In}(\mathrm{OTf})_{3}(10 \mathrm{~mol} \%)$ & reflux & 12 & 7 \\
\hline 5 & $\mathrm{H}_{2} \mathrm{O}$ & $\operatorname{In}(\mathrm{OTf})_{3}(10 \mathrm{~mol} \%)$ & 110 & 12 & trace \\
\hline 6 & - & $\mathrm{In}(\mathrm{OTf})_{3}(10 \mathrm{~mol} \%)$ & 110 & 6 & 53 \\
\hline 7 & - & - & 110 & 10 & 11 \\
\hline 8 & - & $p$-TsOH (10 mol \%) & 110 & 8 & 25 \\
\hline 9 & - & $\mathrm{H}_{2} \mathrm{SO}_{4}(10 \mathrm{~mol} \%)$ & 110 & 8 & 8 \\
\hline 10 & - & $\mathrm{FeCl}_{3}(10 \mathrm{~mol} \%)$ & 110 & 8 & 29 \\
\hline 11 & - & $\mathrm{Sc}(\mathrm{OTf})_{3}(10 \mathrm{~mol} \%)$ & 110 & 8 & 36 \\
\hline 12 & & $\mathrm{InCl}_{3}(10 \mathrm{~mol} \%)$ & 110 & 8 & 42 \\
\hline 13 & - & $\mathrm{In}(\mathrm{OTf})_{3}(10 \mathrm{~mol} \%)$ & 25 & 12 & trace \\
\hline 14 & - & $\operatorname{In}(\mathrm{OTf})_{3}(10 \mathrm{~mol} \%)$ & 100 & 6 & 43 \\
\hline 15 & & $\operatorname{In}(\mathrm{OTf})_{3}(10 \mathrm{~mol} \%)$ & 120 & 6 & 51 \\
\hline 16 & - & $\mathrm{In}(\mathrm{OTf})_{3}(5 \mathrm{~mol} \%)$ & 110 & 6 & 40 \\
\hline 17 & - & $\operatorname{In}(\mathrm{OTf})_{3}(15 \mathrm{~mol} \%)$ & 110 & 6 & 49 \\
\hline 18 & - & $\operatorname{In}(\mathrm{OTf})_{3}(20 \mathrm{~mol} \%)$ & 110 & 6 & 53 \\
\hline
\end{tabular}


Under optimal conditions, we then developed the design and diversity-oriented synthesis of novel 1,4-naphthoquinones possessing indole scaffolds. Different substituted salicylic aldehydes and indoles were applied to this reaction to access structural diversity of target molecules. As illustrated in Table 2, the method is suitable for a wide scope of substituted salicylic aldehydes and indoles. In all cases, the three component reactions are regioselective to 1,4-naphthoquinone derivatives 4 , and their structures were characterized by analytical and spectroscopic methods. For example, the infrared spectra of 4a exhibited that the absorption of two $C=O$ bonds existed at 1650 and $1632 \mathrm{~cm}^{-1}$; the high resolution mass spectrum of $4 \mathbf{a}$ showed the quasi ion peak $\left([\mathrm{M}+\mathrm{Na}]^{+}\right)$at $m / z=476.1262$, which agreed with the 1:1:1 adduct of 2-phenylindole, salicylic aldehyde, and 2-hydroxy-1,4-naphthoquinone with the loss of two water molecules. The ${ }^{1} \mathrm{H}$ NMR spectrum of 4 a displayed two singlets at $\delta=5.82 \mathrm{ppm}$ and $\delta=11.39 \mathrm{ppm}$, which belong to $\mathrm{CH}$ group of $\mathrm{C}-12$ position and $\mathrm{NH}$ protons, respectively. ${ }^{13} \mathrm{C}$ NMR spectrum of $4 \mathbf{a}$ exhibited 29 distinct resonances. Among them, three characteristic signals at $\delta=29.5 \mathrm{ppm}$ (arising from the Ar-CH group), 183.9, and $178.2 \mathrm{ppm}$ (due to the two nonequivalent carbonyl groups) were shown.

Table 2. Preparation of 1,4-naphthoquinones possessing indole scaffolds.

\begin{tabular}{cccccc}
\hline Entry & $\mathbf{R}_{\mathbf{1}}$ & $\mathbf{R}_{\mathbf{2}}$ & Time/h & Product & Yield/\% \\
\hline 1 & $\mathrm{H}$ & $2-\mathrm{Ph}$ & 6 & $\mathbf{4 a}$ & 53 \\
2 & $5-\mathrm{Br}$ & $2-\mathrm{Ph}$ & 5 & $\mathbf{4 b}$ & 57 \\
3 & $5-\mathrm{Cl}$ & $2-\mathrm{Ph}$ & 6 & $\mathbf{4 c}$ & 50 \\
4 & $3-\mathrm{Br}-5-\mathrm{Cl}$ & $2-\mathrm{Ph}$ & 5 & $\mathbf{4 d}$ & 53 \\
5 & $5-\mathrm{Me}$ & $2-\mathrm{Ph}$ & 7 & $\mathbf{4 e}$ & 48 \\
6 & $5-\mathrm{NO} 2$ & $2-\mathrm{Ph}$ & 7 & $\mathbf{4 f}$ & 46 \\
7 & $5-\mathrm{MeO}$ & $2-\mathrm{Ph}$ & 7 & $\mathbf{4 g}$ & 42 \\
8 & $3,5-\mathrm{Br}_{2}$ & $2-\mathrm{Ph}$ & 5 & $\mathbf{4 h}$ & 63 \\
9 & $4-\mathrm{MeO}$ & $2-\mathrm{Ph}$ & 6 & $\mathbf{4 i}$ & 55 \\
10 & $3-\mathrm{Br}-5-\mathrm{Cl}$ & $\mathrm{H}$ & 7 & $\mathbf{4 j}$ & 41 \\
10 & $5-\mathrm{Cl}$ & $\mathrm{H}$ & 7 & $\mathbf{4 k}$ & 42 \\
12 & $5-\mathrm{Br}$ & $\mathrm{H}$ & 7 & $\mathbf{4 1}$ & 44 \\
13 & $5-\mathrm{F}$ & $\mathrm{H}$ & 7 & $\mathbf{4 m}$ & 47 \\
14 & $5-\mathrm{Cl}$ & $2-\mathrm{Me}$ & 5 & $\mathbf{4 n}$ & 59 \\
15 & $5-\mathrm{Me}$ & $2-\mathrm{Me}$ & 5 & $\mathbf{4 o}$ & 48 \\
16 & $\mathrm{H}$ & $2-\mathrm{Me}$ & 5 & $\mathbf{4 p}$ & 50 \\
17 & $\mathrm{H}$ & $5-\mathrm{MeO}$ & 7 & $\mathbf{4 q}$ & 40 \\
18 & $\mathrm{H}$ & $4-\mathrm{MeO}$ & 7 & $\mathbf{4 r}$ & 42 \\
19 & $\mathrm{H}$ & $5-\mathrm{Cl}$ & 7 & $\mathbf{4 s}$ & 43 \\
\hline
\end{tabular}

In Scheme 2, we show the suggested ways to form the hybrid. It is possible that lawone initially reacts with substituted salicylic aldehyde 2 to form olefin $\mathbf{5}$, which goes through a nucleophilic addition of indole to form the Mannich-type intermediate 6 . This step is then followed by an intramolecular dehydration to yield to product 4. 


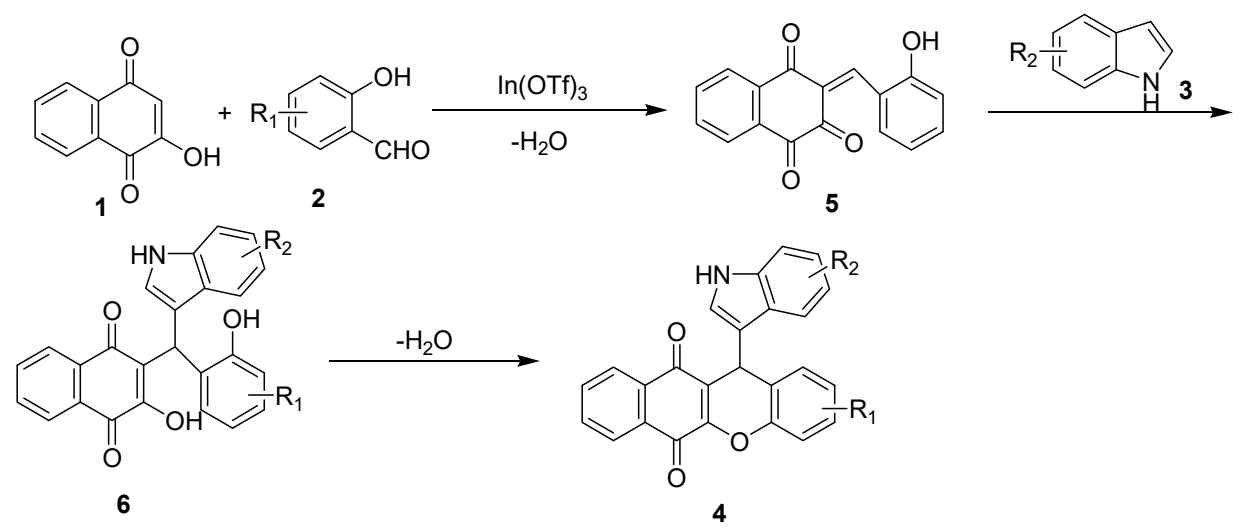

Scheme 2. A suggested pathway for the formation of 1,4-naphthoquinones-possessing indole scaffolds.

\section{Materials and Methods}

\subsection{General Information}

Melting points were determined on a XT-4 binocular microscope and were uncorrected. NMR spectra were determined on Bruker AV-400 spectrometer at room temperature using TMS as internal standard. IR spectra were determined on FTS-40 infrared spectrometer. High resolution mass spectra were recorded on a bruker micrOTOF-QIII mass spectrometer. Commercially available reagents were used throughout without further purification, unless otherwise stated.

\subsection{General Procedure for the Synthesis of Compounds 4}

To a mixture of indole $(1 \mathrm{mmol})$, substituted salicylic aldehyde $(1 \mathrm{mmol})$ and 2-hydroxy-1,4naphthoquinone $(1 \mathrm{mmol}), \mathrm{In}(\mathrm{OTf})_{3}(0.1 \mathrm{mmol})$ was added. The mixture was stirred at $110{ }^{\circ} \mathrm{C}$ for $5-7$ h. After completion of the reaction (TLC), the reaction mixture is cooled to room temperature, treated with water $(10 \mathrm{~mL})$, extracted with $\mathrm{CH}_{2} \mathrm{Cl}_{2}(2 \times 10 \mathrm{~mL})$, and filtered, and the solvent is evaporated in vacuo. Solvent was evaporated and the crude product purified by silica gel column chromatography using petroleum ether: dichloromethane ( $\mathrm{v}: \mathrm{v}=1: 3)$ as eluent to afford the pure product 4 (the copy of IR, ${ }^{1} \mathrm{H}$ NMR, ${ }^{13} \mathrm{C}$ NMR, and HMRS of compounds 4 see Supplementary Materials).

12-(2-Phenyl-1H-indol-3-yl)-12H-benzo[b]xanthene-6,11-dione (4a): Reddish-brown powder, m.p. $260-262{ }^{\circ} \mathrm{C}$, ${ }^{1} \mathrm{H}$ NMR $\left(400 \mathrm{MHz}\right.$,DMSO- $\left.d_{6}\right) \delta: 11.39(\mathrm{~s}, 1 \mathrm{H}), 8.07-8.00(\mathrm{~m}, 3 \mathrm{H}), 7.90-7.80(\mathrm{~m}, 3 \mathrm{H}), 7.59(\mathrm{t}, 2 \mathrm{H}, J=7.6 \mathrm{~Hz})$, $7.49(\mathrm{t}, 1 \mathrm{H}, J=7.2 \mathrm{~Hz}), 7.31-7.28(\mathrm{~m}, 4 \mathrm{H}), 7.23-7.21(\mathrm{~m}, 2 \mathrm{H}), 7.19(\mathrm{t}, 2 \mathrm{H}, J=1.2 \mathrm{~Hz}), 5.82(\mathrm{~s}, 1 \mathrm{H}) ;{ }^{13} \mathrm{C} \mathrm{NMR}$ (100 MHz, DMSO-d6) $\delta: 183.9,178.2,150.9,148.2,136.3,135.3,135.2,134.4,133.4,131.8,130.7,129.8,129.6$ (2C), 129.2 (2C), 128.7, 128.6, 126.9, 126.5, 126.3, 126.1, 124.1, 121.8, 121.3, 119.8, 118.1, 117.2, 115.2, 112.0, 29.5; IR (KBr): $v$ 3360, 1632, $1650 \mathrm{~cm}^{-1}$; HRMS-ESI $(m / z)$ : calcd for $\mathrm{C}_{31} \mathrm{H}_{19} \mathrm{NNaO}_{3}[\mathrm{M}+\mathrm{Na}]^{+}: 476.1263$, found: 476.1262 .

2-Bromo-12-(2-Phenyl-1H-indol-3-yl)-12H-benzo[b]xanthene-6,11-dione (4b): Red powder, m.p. $279-280{ }^{\circ} \mathrm{C}$, ${ }^{1} \mathrm{H}$ NMR $\left(400 \mathrm{MHz}, \mathrm{DMSO}-d_{6}\right) \delta: 11.46(\mathrm{~s}, 1 \mathrm{H}), 8.04-8.02(\mathrm{~m}, 1 \mathrm{H}), 7.93-7.78(\mathrm{~m}, 5 \mathrm{H}), 7.58(\mathrm{t}, J=7.6 \mathrm{~Hz}$, $2 \mathrm{H}), 7.48(\mathrm{t}, J=7.2 \mathrm{~Hz}, 1 \mathrm{H}), 7.37-7.32(\mathrm{~m}, 3 \mathrm{H}), 7.24(\mathrm{~d}, J=8.8 \mathrm{~Hz}, 1 \mathrm{H}), 7.04(\mathrm{t}, J=7.6 \mathrm{~Hz}, 1 \mathrm{H}), 6.92-6.89$ (m, 2H), 5.79 (s, 1H); ${ }^{13} \mathrm{C}$ NMR (100 MHz, DMSO-d6) $\delta: 183.6,177.8,150.5,147.3,136.2,135.6,135.2$, 134.5, 133.2, 132.0, 131.6 (2C), 130.6, 129.5 (2C), 129.3 (2C), 128.7, 126.8, 126.6, 126.5, 126.3, 122.0, 120.9, 120.0, 119.6, 118.0, 117.4, 114.8, 112.2, 19.4; IR (KBr): $v 3379,1671,1634 \mathrm{~cm}^{-1}$; HRMS-ESI $(\mathrm{m} / \mathrm{z})$ : calcd for $\mathrm{C}_{31} \mathrm{H}_{18} \mathrm{BrNNaO}_{3}[\mathrm{M}+\mathrm{Na}]^{+}: 554.0368$, found: 554.0362 .

2-Chloro-12-(2-Phenyl-1H-indol-3-yl)-12H-benzo[b]xanthene-6,11-dione (4c): Red powder, m.p. 275-276 ${ }^{\circ} \mathrm{C}$, ${ }^{1} \mathrm{H}$ NMR $\left(400 \mathrm{MHz}, \mathrm{DMSO}-d_{6}\right) \delta: 11.46(\mathrm{~s}, 1 \mathrm{H}), 8.05-8.03(\mathrm{~m}, 1 \mathrm{H}), 7.93-7.80(\mathrm{~m}, 5 \mathrm{H}), 7.58(\mathrm{t}, J=7.2 \mathrm{~Hz}$, 
2H), $7.49(\mathrm{t}, J=7.2 \mathrm{~Hz}, 1 \mathrm{H}), 7.34-7.32(\mathrm{~m}, 3 \mathrm{H}), 7.27-7.24(\mathrm{~m}, 1 \mathrm{H}), 7.04(\mathrm{~d}, J=7.6 \mathrm{~Hz}, 1 \mathrm{H}), 6.92(\mathrm{t}$, $J=7.6 \mathrm{~Hz}, 1 \mathrm{H}), 6.77(\mathrm{~d}, J=2.0 \mathrm{~Hz}, 1 \mathrm{H}), 6.92-6.89(\mathrm{~m}, 2 \mathrm{H}), 5.81(\mathrm{~s}, 1 \mathrm{H}) ;{ }^{13} \mathrm{C}$ NMR (100 MHz, DMSO-d6) $\delta: 183.6,177.9,150.6,146.9,136.2,135.6,135.2,134.5,133.2,131.7,130.7,129.5$ (2C), 129.4 (2C), 129.3, 129.0, 128.8, 128.7, 126.8, 126.5, 126.3, 126.2, 122.0, 120.8, 120.0, 119.3, 118.0, 114.8, 112.2, 29.6; IR (KBr): $v$ 3377, 1675, $1634 \mathrm{~cm}^{-1}$; HRMS-ESI $(m / z)$ : calcd for $\mathrm{C}_{31} \mathrm{H}_{18} \mathrm{ClNNaO}_{3}[\mathrm{M}+\mathrm{Na}]^{+}: 510.0873$, found: 510.0858 .

4-Bromo-2-chloro-12-(2-Phenyl-1H-indol-3-yl)-12H-benzo[b]xanthene-6,11-dione (4d): Red powder, m.p. 243-244 ${ }^{\circ} \mathrm{C},{ }^{1} \mathrm{H}$ NMR $\left(400 \mathrm{MHz}, \mathrm{DMSO}-d_{6}\right) \delta: 11.49(\mathrm{~s}, 1 \mathrm{H}), 8.07-8.04(\mathrm{~m}, 1 \mathrm{H}), 7.90-7.80(\mathrm{~m}, 5 \mathrm{H}), 7.67$ $(\mathrm{d}, J=2.4 \mathrm{~Hz}, 1 \mathrm{H}), 7.57-7.46(\mathrm{~m}, 3 \mathrm{H}), 7.39-7.32(\mathrm{~m}, 2 \mathrm{H}), 7.08-7.04(\mathrm{~m}, 1 \mathrm{H}), 6.97-6.93(\mathrm{~m}, 1 \mathrm{H}), 6.78(\mathrm{~d}$, $J=2.4 \mathrm{~Hz}, 1 \mathrm{H}), 5.85(\mathrm{~s}, 1 \mathrm{H}) ;{ }^{13} \mathrm{C}$ NMR $(100 \mathrm{MHz}$, DMSO-d6) $\delta: 183.5,177.4,150.3,144.2,136.1,136.0$, 135.2, 134.6, 133.0, 131.6, 130.7, 129.6, 129.5 (2C), 129.2 (2C), 128.8, 128.5, 127.7, 127.4, 126.8, 126.5, 126.4, 122.1, 121.3, 120.1, 118.0, 114.6, 112.2, 112.0, 30.0; IR (KBr): $v$ 3288, 1685, $1637 \mathrm{~cm}^{-1}$; HRMS-ESI $(\mathrm{m} / \mathrm{z})$ : calcd for $\mathrm{C}_{31} \mathrm{H}_{17} \mathrm{BrClNNaO}_{3}[\mathrm{M}+\mathrm{Na}]^{+}$: 587.9978, found: 587.9968 .

2-Methyl-12-(2-Phenyl-1H-indol-3-yl)-12H-benzo[b]xanthene-6,11-dione (4e): Orange-red powder, m.p. 244-245 ${ }^{\circ} \mathrm{C},{ }^{1} \mathrm{H}$ NMR $\left(400 \mathrm{MHz}, \mathrm{DMSO}-d_{6}\right) \delta: 11.38(\mathrm{~s}, 1 \mathrm{H}), 8.03-7.99(\mathrm{~m}, 3 \mathrm{H}), 7.86-776(\mathrm{~m}, 3 \mathrm{H})$, $7.60(\mathrm{t}, J=7.6 \mathrm{~Hz}, 2 \mathrm{H}), 7.50(\mathrm{t}, J=7.2 \mathrm{~Hz}, 1 \mathrm{H}), 7.32-7.26(\mathrm{~m}, 2 \mathrm{H}), 7.13(\mathrm{~d}, J=8.0 \mathrm{~Hz}, 1 \mathrm{H}), 7.03-6.96$ $(\mathrm{m}, 2 \mathrm{H}), 6.86(\mathrm{t}, J=7.2 \mathrm{~Hz}, 2 \mathrm{H}), 6.63(\mathrm{~s}, 3 \mathrm{H}), 5.72(\mathrm{~s}, 1 \mathrm{H}) ;{ }^{13} \mathrm{C}$ NMR (100 MHz, DMSO-d6) $\delta: 183.7$, 178.2, 150.9, 146.2, 136.3, 135.3, 135.1, 134.3, 133.5, 131.8, 130.7, 129.8, 129.5 (2C), 129.4 (2C), 129.2, $128.5,126.9,126.4,126.3,123.7,121.8,121.1,119.7,118.1,117.0,115.2,112.0,29.5,20.8$; IR (KBr): $v$ $3372,1677,1631 \mathrm{~cm}^{-1}$; HRMS-ESI $(m / z)$ : calcd for $\mathrm{C}_{32} \mathrm{H}_{21} \mathrm{NNaO}_{3}[\mathrm{M}+\mathrm{Na}]^{+}: 490.1419$, found: 490.1410 .

2-Nitro-12-(2-Phenyl-1H-indol-3-yl)-12H-benzo[b]xanthene-6,11-dione (4f): Yellow powder, m.p. 245-246 ${ }^{\circ} \mathrm{C},{ }^{1} \mathrm{H}$ NMR $\left(400 \mathrm{MHz}\right.$, DMSO- $\left.d_{6}\right) \delta$ : $11.49(\mathrm{~s}, 1 \mathrm{H}), 8.07-8.04(\mathrm{~m}, 1 \mathrm{H}), 7.90-7.80(\mathrm{~m}$, $5 \mathrm{H}), 7.67(\mathrm{~d}, J=2.4 \mathrm{~Hz}, 1 \mathrm{H}), 7.57-7.46(\mathrm{~m}, 3 \mathrm{H}), 7.39-7.32(\mathrm{~m}, 2 \mathrm{H}), 7.08-7.04(\mathrm{~m}, 1 \mathrm{H}), 6.97-6.93$ $(\mathrm{m}, 1 \mathrm{H}), 6.78(\mathrm{~d} J=2.4 \mathrm{~Hz}, 1 \mathrm{H}), 5.85(\mathrm{~s}, 1 \mathrm{H}) ;{ }^{13} \mathrm{C}$ NMR $(100 \mathrm{MHz}$, DMSO-d6) $\delta:$ 183.6, 177.6, 152.3, 150.2, 144.7, 136.2, 136.0, 135.3, 134.7, 133.0, 131.6, 130.7, 129.5 (2C), 129.3 (2C), 128.9, 126.8, 126.6, 126.4, 125.7, 125.5, 124.4, 122.1, 121.3, 120.1, 118.7, 118.1, 114.9, 112.2, 29.6; IR (KBr): $v$ 3358, 1680, 1665, $1644 \mathrm{~cm}^{-1}$; HRMS-ESI $(\mathrm{m} / z)$ : calcd for $\mathrm{C}_{31} \mathrm{H}_{18} \mathrm{~N}_{2} \mathrm{NaO}_{5}[\mathrm{M}+\mathrm{Na}]^{+}: 521.113$, found: 521.1106.

2-Methoxy-12-(2-Phenyl-1H-indol-3-yl)-12H-benzo[b]xanthene-6,11-dione (4g): Yellow powder, m.p. 211-212 ${ }^{\circ} \mathrm{C},{ }^{1} \mathrm{H}$ NMR $\left(400 \mathrm{MHz}, \mathrm{DMSO}-d_{6}\right) \delta: 11.35(\mathrm{~s}, 1 \mathrm{H}), 8.06-7.98(\mathrm{~m}, 3 \mathrm{H}), 7.88-7.78(\mathrm{~m}, 3 \mathrm{H}), 7.59$ $(\mathrm{d}, J=7.6 \mathrm{~Hz}, 2 \mathrm{H}), 7.48(\mathrm{~d}, J=7.6 \mathrm{~Hz}, 1 \mathrm{H}), 7.31-7.26(\mathrm{~m}, 2 \mathrm{H}), 7.02-6.98(\mathrm{~m}, 1 \mathrm{H}), 6.88-6.83(\mathrm{~m}, 2 \mathrm{H})$, $6.73(\mathrm{~d} J=7.4 \mathrm{~Hz}, 1 \mathrm{H}), 6.60-6.58(\mathrm{~m}, 1 \mathrm{H}), 5.72(\mathrm{~s}, 1 \mathrm{H}), 3.71(\mathrm{~s}, 3 \mathrm{H}) ;{ }^{13} \mathrm{C}$ NMR $(100 \mathrm{MHz}$, DMSO-d6) $\delta$ : 183.8, 178.2, 159.4, 150.9, 148.7, 136.3, 135.2, 135.1, 134.4, 133.5, 131.8, 130.7, 130.3, 129.5 (2C), 129.2 (2C), $128.5,126.9,126.4,126.3,121.8,121.6,119.7,118.2,115.9,115.4,113.2,112.0,101.7,55.9,29.0 ; \operatorname{IR~}(\mathrm{KBr}): v$ 3326, 1682, $1638 \mathrm{~cm}^{-1}$; HRMS-ESI $(m / z)$ : calcd for $\mathrm{C}_{32} \mathrm{H}_{21} \mathrm{NNaO}_{4}[\mathrm{M}+\mathrm{Na}]^{+}: 506.1368$, found: 506.1358 .

2,4-Dibromo-12-(2-phenyl-1H-indol-3-yl)-12H-benzo[b]xanthene-6,11-dione (4h): Red powder, m.p. 252-253 ${ }^{\circ} \mathrm{C},{ }^{1} \mathrm{H}$ NMR $\left(400 \mathrm{MHz}, \mathrm{DMSO}-d_{6}\right) \delta: 11.50(\mathrm{~s}, 1 \mathrm{H}), 8.06(\mathrm{dd}, J=2.0,7.6 \mathrm{~Hz}, 1 \mathrm{H}), 7.88-7.82(\mathrm{~m}$, $5 \mathrm{H}), 7.77(\mathrm{~d}, J=2.4 \mathrm{~Hz}, 1 \mathrm{H}), 7.57-7.47(\mathrm{~m}, 3 \mathrm{H}), 7.39-7.33(\mathrm{~m}, 2 \mathrm{H}), 7.08-7.04(\mathrm{~m}, 1 \mathrm{H}), 6.96(\mathrm{t}, J=7.6 \mathrm{~Hz}$, $1 \mathrm{H}), 6.90(\mathrm{~d}, J=7.0 \mathrm{~Hz}, 1 \mathrm{H}), 5.85(\mathrm{~s}, 1 \mathrm{H}) ;{ }^{13} \mathrm{C}$ NMR (100 MHz, DMSO-d6) $\delta: 183.5,177.4,150.3,144.6$, 136.1, 136.0, 135.2, 134.6, 134.2, 133.0, 131.6, 131.5, 130.7, 129.5 (2C), 129.3 (2C), 128.8, 128.1, 126.8, 126.5, 126.4, 122.1, 121.4, 120.1, 118.0, 117.3, 114.7, 112.2, 112.1, 29.9; IR (KBr): v 3289, 1683, $1635 \mathrm{~cm}^{-1}$; HRMS-ESI $(m / z)$ : calcd for $\mathrm{C}_{31} \mathrm{H}_{17} \mathrm{Br}_{2} \mathrm{NNaO}_{3}[\mathrm{M}+\mathrm{Na}]^{+}$: 631.9473, found: 631.9462 .

3-Methoxy-12-(2-phenyl-1H-indol-3-yl)-12H-benzo[b]xanthene-6,11-dione (4i): Orange-red powder, m.p. 244-245 ${ }^{\circ} \mathrm{C},{ }^{1} \mathrm{H}$ NMR $\left(400 \mathrm{MHz}, \mathrm{DMSO}-d_{6}\right) \delta: 11.40(\mathrm{~s}, 1 \mathrm{H}), 8.05-8.00(\mathrm{~m}, 3 \mathrm{H}), 7.88-7.78(\mathrm{~m}, 3 \mathrm{H}), 7.61$ $(\mathrm{d}, J=7.6 \mathrm{~Hz}, 2 \mathrm{H}), 7.50(\mathrm{t}, J=7.6 \mathrm{~Hz}, 1 \mathrm{H}), 7.32-7.20(\mathrm{~m}, 3 \mathrm{H}), 7.03-6.99(\mathrm{~m}, 1 \mathrm{H}), 6.88-6.84(\mathrm{~m}, 1 \mathrm{H})$, 
$6.77(\mathrm{dd}, J=2.8,8.8 \mathrm{~Hz}, 1 \mathrm{H}), 5.75(\mathrm{~s}, 1 \mathrm{H}), 3.51(\mathrm{~s}, 1 \mathrm{H}) ;{ }^{13} \mathrm{C}$ NMR (100 MHz, DMSO-d6) $\delta: 183.8,178.2$, 156.8, 151.2, 142.1, 136.3, 135.3, 135.1, 134.3, 133.4, 131.8, 130.7, 129.6 (2C), 129.3 (2C), 128.6, 126.8, 126.4, 126.3, 125.1, 121.8, 120.2, 119.7, 118.3, 118.1, 115.0, 114.1, 114.0, 112.1, 55.5, 29.9; IR (KBr): v $3304,1683,1638 \mathrm{~cm}^{-1}$; HRMS-ESI $(\mathrm{m} / z)$ : calcd for $\mathrm{C}_{32} \mathrm{H}_{21} \mathrm{NNaO}_{4}[\mathrm{M}+\mathrm{Na}]^{+}: 506.1368$, found: 506.1357 .

4-Bromo-2-chloro-12-(1H-indol-3-yl)-12H-benzo[b]xanthene-6,11-dione (4j): Yellow-brown powder, m.p. 248-250 ${ }^{\circ} \mathrm{C},{ }^{1} \mathrm{H}$ NMR $\left(400 \mathrm{MHz}\right.$, DMSO- $\left.d_{6}\right) \delta: 11.08(\mathrm{~s}, 1 \mathrm{H}), 8.10(\mathrm{~d}, J=7.2 \mathrm{~Hz}, 1 \mathrm{H}), 7.92-7.84(\mathrm{~m}, 3 \mathrm{H})$, $7.73(\mathrm{~s}, 1 \mathrm{H}), 7.68(\mathrm{~d}, J=8.0 \mathrm{~Hz}, 1 \mathrm{H}), 7.52(\mathrm{~s}, 1 \mathrm{H}), 7.38(\mathrm{~s}, 1 \mathrm{H}), 7.33-7.31(\mathrm{~d}, J=7.6 \mathrm{~Hz}, 1 \mathrm{H}), 7.08-7.00(\mathrm{~m}$, 2H), $5.71(\mathrm{~s}, 1 \mathrm{H}) ;{ }^{13} \mathrm{C}$ NMR $\left(100 \mathrm{MHz}\right.$, DMSO- $\left.d_{6}\right) \delta: 183.2,177.7,150.5,144.9,136.7,135.0,134.6,131.7$, 131.3, 131.2, 129.6, 129.0, 128.6, 126.6, 126.2, 125.5, 124.9, 122.1, 121.8, 119.7, 118.5, 118.2, 112.3, 111.8, 29.9; IR (KBr): $v$ 3403, 1633, $1680 \mathrm{~cm}^{-1}$; HRMS-ESI $(\mathrm{m} / \mathrm{z})$ : calcd for $\mathrm{C}_{25} \mathrm{H}_{13} \mathrm{BrClNNaO}_{3}[\mathrm{M}+\mathrm{Na}]^{+}$: 511.9665, found: 511.9664 .

2-chloro-12-(1H-indol-3-yl)-12H-benzo[b]xanthene-6,11-dione (4k): Brown powder, m.p. $343-345{ }^{\circ} \mathrm{C}$, ${ }^{1} \mathrm{H}$ NMR $\left(400 \mathrm{MHz}\right.$, DMSO- $\left.d_{6}\right) \delta: 11.04(\mathrm{~s}, 1 \mathrm{H}), 7.91-7.89(\mathrm{~m}, 1 \mathrm{H}), 7.85-7.82(\mathrm{~m}, 3 \mathrm{H}), 7.61(\mathrm{~d}, J=8.0 \mathrm{~Hz}$, 1H), 7.46-7.30 (m, 5H), 7.06-6.96 (m, 2H), $5.67(\mathrm{~s}, 1 \mathrm{H}) ;{ }^{13} \mathrm{C}$ NMR (100 MHz, DMSO- $\left.d_{6}\right) \delta: 183.4,178.2$, 150.5, 147.6, 136.7, 135.0, 134.4, 131.8, 131.1, 129.5, 129.4, 128.5, 127.1, 126.5, 126.2, 125.6, 124.6, 121.7, $119.5,119.3,118.5(2 \mathrm{C}), 112.3,29.5$; IR (KBr): $v$ 3410, 1634, $1685 \mathrm{~cm}^{-1}$; HRMS-ESI $(m / z)$ : calcd for $\mathrm{C}_{25} \mathrm{H}_{14} \mathrm{ClNNaO}_{3}[\mathrm{M}+\mathrm{Na}]^{+}:$434.0560, found: 434.0559 .

2-Bromo-12-(1H-indol-3-yl)-12H-benzo[b]xanthene-6,11-dione (41): Reddish-brown powder, m.p. 262-264 ${ }^{\circ} \mathrm{C}$, ${ }^{1} \mathrm{H}$ NMR $\left(400 \mathrm{MHz}\right.$, DMSO- $\left.d_{6}\right) \delta: 8.10-8.07(\mathrm{~m}, 1 \mathrm{H}), 7.91-7.82(\mathrm{~m}, 3 \mathrm{H}), 7.61-7.58(\mathrm{~m}, 2 \mathrm{H}), 7.46-7.43(\mathrm{~m}$, $1 \mathrm{H}), 7.36-7.30(\mathrm{~m}, 3 \mathrm{H}), 7.04(\mathrm{t}, J=7.2 \mathrm{~Hz}, 1 \mathrm{H}), 6.98(\mathrm{t}, J=7.6 \mathrm{~Hz}, 1 \mathrm{H}) ;{ }^{13} \mathrm{C}$ NMR $\left(100 \mathrm{MHz}\right.$, DMSO- $\left.d_{6}\right) \delta$ : 183.3, 178.2, 150.5, 148.1, 136.7, 135.0, 134.4, 132.5, 131.8, 131.4, 131.1, 127.5, 126.5, 126.2, 125.6, 124.6, 121.8, 121.7, 119.7, 119.5 (2C), 118.5, 117.4, 112.3, 29.4; IR (KBr): $v 3410,1633,1683 \mathrm{~cm}^{-1}$; HRMS-ESI $(\mathrm{m} / \mathrm{z})$ : calcd for $\mathrm{C}_{25} \mathrm{H}_{14} \mathrm{BrNNaO}_{3}[\mathrm{M}+\mathrm{Na}]^{+}: 478.0055$, found: 478.0056 .

2-Fluoro-12-(1H-indol-3-yl)-12H-benzo[b]xanthene-6,11-dione (4m): Reddish-brown powder, m.p. 330-332 ${ }^{\circ} \mathrm{C}$, ${ }^{1} \mathrm{H}$ NMR $\left(400 \mathrm{MHz}\right.$, DMSO- $\left.d_{6}\right) \delta: 11.02(\mathrm{~s}, 1 \mathrm{H}), 8.10-8.08(\mathrm{~m}, 1 \mathrm{H}), 7.92-7.82(\mathrm{~m}, 3 \mathrm{H}), 7.61(\mathrm{~d}, J=8.8 \mathrm{~Hz}$, $1 \mathrm{H}), 7.43-7.40(\mathrm{~m}, 1 \mathrm{H}), 7.35-7.22(\mathrm{~m}, 3 \mathrm{H}), 7.15-7.10(\mathrm{~m}, 1 \mathrm{H}), 7.04(\mathrm{t}, J=7.2 \mathrm{~Hz}, 1 \mathrm{H}), 6.97(\mathrm{t}, J=6.8 \mathrm{~Hz}, 1 \mathrm{H})$, $5.66(\mathrm{~s}, 1 \mathrm{H}) ;{ }^{13} \mathrm{C}$ NMR $\left(100 \mathrm{MHz}\right.$, DMSO- $\left.d_{6}\right) \delta: 183.4,178.4,160.6,150.7,145.2,136.8,135.0,134.4,131.8$, 131.1, 126.5, 126.2, 125.6, 124.5, 121.6, 121.2, 119.5, 119.2, 119.1, 118.5, 118.4, 115.8, 115.7, 112.2, 29.8; IR (KBr): $v 3416,1650,1638 \mathrm{~cm}^{-1}$; HRMS-ESI $(\mathrm{m} / \mathrm{z})$ : calcd for $\mathrm{C}_{25} \mathrm{H}_{14} \mathrm{FNNaO}_{3}[\mathrm{M}+\mathrm{Na}]^{+}: 418.0855$, found: 418.0858 .

2-Chloro-12-(2-methyl-1H-indol-3-yl)-12H-benzo[b]xanthene-6,11-dione (4n): Reddish-brown powder, m.p. 264-265 ${ }^{\circ} \mathrm{C},{ }^{1} \mathrm{H}$ NMR $\left(400 \mathrm{MHz}\right.$, DMSO- $\left.d_{6}\right) \delta: 11.00(\mathrm{~s}, 1 \mathrm{H}), 8.05-7.79(\mathrm{~m}, 4 \mathrm{H}), 7.41(\mathrm{~d}, J=8.8 \mathrm{~Hz}, 1 \mathrm{H})$, 7.33-7.30 (m, 1H), $7.19(\mathrm{~d}, J=7.6 \mathrm{~Hz}, 2 \mathrm{H}), 7.07(\mathrm{~d}, J=7.6 \mathrm{~Hz}, 1 \mathrm{H}), 6.88(\mathrm{~d}, J=7.2 \mathrm{~Hz}, 1 \mathrm{H}), 6.79(\mathrm{~d}$, $J=7.2 \mathrm{~Hz}, 1 \mathrm{H}), 5.57(\mathrm{~s}, 1 \mathrm{H}), 2.63(\mathrm{~s}, 3 \mathrm{H}) ;{ }^{13} \mathrm{C}$ NMR $\left(100 \mathrm{MHz}, \mathrm{DMSO}-d_{6}\right) \delta: 183.4,178.2,149.9,147.8$, 135.5, 135.1, 134.5, 133.4, 131.7, 130.8, 129.9, 129.6, 128.7, 126.7, 126.5, 126.2, 121.1, 120.5, 119.3, 119.0, 116.9, 113.5, 111.4, 29.1, 12.1; IR (KBr): $v 3379,1679,1660,1635 \mathrm{~cm}^{-1}$; HRMS-ESI $(\mathrm{m} / \mathrm{z})$ : calcd for $\mathrm{C}_{26} \mathrm{H}_{16} \mathrm{ClNNaO}_{3}[\mathrm{M}+\mathrm{Na}]^{+}$: 448.0716, found: 448.0707 .

2-Methyl-12-(2-methyl-1H-indol-3-yl)-12H-benzo[b]xanthene-6,11-dione (4o): Reddish-brown powder, m.p. 269-270 ${ }^{\circ} \mathrm{C},{ }^{1} \mathrm{H}$ NMR $\left(400 \mathrm{MHz}\right.$, DMSO- $\left.d_{6}\right) \delta: 10.92(\mathrm{~s}, 1 \mathrm{H}), 8.01(\mathrm{dd}, J=4.0,7.2 \mathrm{~Hz}, 1 \mathrm{H}), 7.85-7.75(\mathrm{~m}$, $3 \mathrm{H}), 7.20-7.17(\mathrm{~m}, 2 \mathrm{H}), 7.09-7.02(\mathrm{~m}, 2 \mathrm{H}), 6.90-6.85(\mathrm{~m}, 2 \mathrm{H}), 6.77(\mathrm{t}, J=7.6 \mathrm{~Hz}, 1 \mathrm{H}), 5.44(\mathrm{~s}, 1 \mathrm{H}), 2.59$ (s, 3H), 2.15 (s, 3H); ${ }^{13} \mathrm{C}$ NMR (100 MHz, DMSO-d $\left.d_{6}\right) \delta: 183.5,178.5,150.2,146.9,135.4,135.3,135.0$, 134.3, 132.9, 131.7, 130.8, 130.4, 129.3, 126.8, 126.4, 126.1, 123.6, 121.4, 120.4, 119.1, 117.1, 116.8, 114.1, 111.2, 29.1, 20.7, 12.1; IR (KBr): $v$ 3375, 1673, $1632 \mathrm{~cm}^{-1}$; HRMS-ESI $(m / z)$ : calcd for $\mathrm{C}_{27} \mathrm{H}_{19} \mathrm{NNaO}_{3}$ $[\mathrm{M}+\mathrm{Na}]^{+}:$428.1263, found: 428.1263 . 
12-(2-Methyl-1H-indol-3-yl)-12H-benzo[b]xanthene-6,11-dione (4p): Yellow-brown powder, m.p. $350-352{ }^{\circ} \mathrm{C}$, ${ }^{1} \mathrm{H}$ NMR $\left(400 \mathrm{MHz}\right.$, DMSO- $\left.d_{6}\right) \delta: 10.94(\mathrm{~s}, 1 \mathrm{H}), 8.05(\mathrm{~d}, J=4.4 \mathrm{~Hz}, 1 \mathrm{H}), 7.86-7.80(\mathrm{~m}, 3 \mathrm{H}), 7.35-7.24(\mathrm{~m}$, 2H), 7.18-7.07 (m, 4H), $6.87(\mathrm{t}, J=7.2 \mathrm{~Hz}, 1 \mathrm{H}), 6.77(\mathrm{t}, J=7.2 \mathrm{~Hz}, 1 \mathrm{H}) 5.55(\mathrm{~s}, 1 \mathrm{H}) ;{ }^{13} \mathrm{C} \mathrm{NMR}(100 \mathrm{MHz}$, DMSO- $\left.d_{6}\right) \delta: 183.5,178.5,150.2,148.9,135.5,135.1,134.4,132.9,131.7,130.8,130.5,128.6,126.8,126.5$, 126.2, 126.1, 124.0, 121.6, 120.4, 119.1, 117.1, 117.0, 114.1, 111.2, 29.0, 12.1; IR (KBr): v 3401, 2919, 1631, $1649 \mathrm{~cm}^{-1}$; HRMS-ESI $(m / z)$ : calcd for $\mathrm{C}_{26} \mathrm{H}_{17} \mathrm{NNaO}_{3}[\mathrm{M}+\mathrm{Na}]^{+}: 414.1106$, found: 414.1102 .

12-(5-Methoxy-1H-indol-3-yl)-12H-benzo[b]xanthene-6,11-dione (4q): Yellow-brown powder, m.p. $260-261{ }^{\circ} \mathrm{C}$, ${ }^{1} \mathrm{H}$ NMR $\left(400 \mathrm{MHz}\right.$, DMSO- $\left.d_{6}\right) \delta: 10.82(\mathrm{~s}, 1 \mathrm{H}), 8.10-8.08(\mathrm{~m}, 1 \mathrm{H}), 7.93-7.83(\mathrm{~m}, 3 \mathrm{H}), 7.42(\mathrm{~d}, J=7.2 \mathrm{~Hz}$, $1 \mathrm{H}), 7.35(\mathrm{~d}, J=7.6 \mathrm{~Hz}, 1 \mathrm{H}), 7.30-7.24(\mathrm{~m}, 2 \mathrm{H}), 7.19-7.12(\mathrm{~m}, 2 \mathrm{H}), 7.06(\mathrm{~d}, J=2.0 \mathrm{~Hz}, 1 \mathrm{H}), 6.70-6.67(\mathrm{~m}$, 1H), $5.61(\mathrm{~s}, 1 \mathrm{H}), 3.72(\mathrm{~s}, 3 \mathrm{H}) ;{ }^{13} \mathrm{C}$ NMR $\left(100 \mathrm{MHz}, \mathrm{DMSO}-d_{6}\right) \delta: 183.5,178.5,153.7,150.7,149.0,135.0$, 134.4, 131.9, 131.1, 130.2, 128.6, 126.8, 126.5, 126.2, 126.0, 125.0, 124.9, 122.2, 118.7, 117.2, 112.8, 111.3, 100.7, 55.7, 29.5; IR (KBr): $v 3442,2926,1631,1654 \mathrm{~cm}^{-1}$; HRMS-ESI $(m / z)$ : calcd for $\mathrm{C}_{26} \mathrm{H}_{17} \mathrm{NNaO}_{4}[\mathrm{M}+\mathrm{Na}]^{+}$: 430.1055, found: 430.1062 .

12-(4-Methoxy-1H-indol-3-yl)-12H-benzo[b]xanthene-6,11-dione (4r): Yellow-brown powder, m.p. 190-192 ${ }^{\circ} \mathrm{C}$, ${ }^{1} \mathrm{H}$ NMR $\left(400 \mathrm{MHz}\right.$, DMSO- $\left.d_{6}\right) \delta: 8.12-8.10(\mathrm{~m}, 1 \mathrm{H}), 7.87-7.81(\mathrm{~m}, 3 \mathrm{H}), 7.47(\mathrm{~d}, J=7.6 \mathrm{~Hz}, 1 \mathrm{H}), 7.27-7.16$ $(\mathrm{m}, 3 \mathrm{H}), 7.09(\mathrm{t}, J=6.4 \mathrm{~Hz}, 1 \mathrm{H}), 6.97(\mathrm{t}, J=7.6 \mathrm{~Hz}, 1 \mathrm{H}), 6.89(\mathrm{~d}, J=8.0 \mathrm{~Hz}, 1 \mathrm{H}), 6.51(\mathrm{~d}, J=7.6 \mathrm{~Hz}, 1 \mathrm{H}), 5.91$ $(\mathrm{s}, 1 \mathrm{H}), 3.94(\mathrm{~s}, 3 \mathrm{H}) ;{ }^{13} \mathrm{C}$ NMR $\left(100 \mathrm{MHz}, \mathrm{DMSO}-d_{6}\right) \delta: 183.6,178.7,154.2,151.0,148.4,138.3,135.0(2 \mathrm{C})$, 133.7, 132.4, 131.1, 130.1, 128.0, 126.4, 125.9, 123.4, 122.7, 120.1, 117.2, 115.7, 111.5, 105.5, 99.9, 55.4, 29.8; IR(KBr): $v$ 3441, 2930, 1633, $1644 \mathrm{~cm}^{-1}$; HRMS-ESI $(m / z)$ : calcd for $\mathrm{C}_{26} \mathrm{H}_{17} \mathrm{NNaO}_{4}[\mathrm{M}+\mathrm{Na}]^{+}: 430.1055$, found: 430.1054 .

12-(5-Chloro-1H-indol-3-yl)-12H-benzo[b]xanthene-6,11-dione (4s): Yellow-brown powder, m.p. $256-257^{\circ} \mathrm{C}$, ${ }^{1} \mathrm{H}$ NMR $\left(400 \mathrm{MHz}\right.$, DMSO- $\left.d_{6}\right) \delta: 11.19(\mathrm{~s}, 1 \mathrm{H}), 8.10-8.08(\mathrm{~m}, 1 \mathrm{H}), 7.93-7.83(\mathrm{~m}, 3 \mathrm{H}), 7.72(\mathrm{~d}, J=1.6 \mathrm{~Hz}$, 1H), 7.16-7.12 (m, 5H), $7.06(\mathrm{t}, J=2.0 \mathrm{~Hz}, 1 \mathrm{H}), 7.03(\mathrm{~d}, J=2.0 \mathrm{~Hz}, 1 \mathrm{H}), 5.66(\mathrm{~s}, 1 \mathrm{H}) ;{ }^{13} \mathrm{C} \mathrm{NMR}(100 \mathrm{MHz}$, DMSO- $\left.d_{6}\right) \delta: 183.5,178.4,150.8,148.8,135.1,134.9,134.4,131.8,131.2,130.2,128.7,126.9,126.5,126.3$, 126.2, 126.1, 124.9, 124.1, 122.1, 121.6, 119.3, 118.1, 117.3, 113.7, 29.1; IR(KBr): 3407, 2922, 1633, $1685 \mathrm{~cm}^{-1}$; HRMS-ESI $(m / z)$ : calcd for $\mathrm{C}_{25} \mathrm{H}_{14} \mathrm{ClNNaO}_{3}[\mathrm{M}+\mathrm{Na}]^{+}$: 434.0560, found: 434.0557 .

\section{Conclusions}

A green and efficient procedure for the synthesis of 1,4-naphthoquinones possessing indole scaffolds was investigated via the three-component reaction of 2-hydroxy-1,4-naphthoquinone, substituted salicylic aldehydes, and indoles using $\operatorname{In}(\mathrm{OTf})_{3}$ as a catalyst. The method has the advantages of simple operation, mild reaction conditions, and a hospitable environment.

Supplementary Materials: The following are available online: IR, ${ }^{1} \mathrm{H} \mathrm{NMR},{ }^{13} \mathrm{C}$ NMR, and HMRS of compounds 4 . Author Contributions: L.W. conceived and designed the experiments; X.Y. performed the synthesis.

Funding: This research was funded by NSFC-Henan Joint Fund (No. U1604164) and Key Scientific Research Projects in Universities of Henan Province (No. 16A350010).

Acknowledgments: We are grateful for financial support from the Natural Science Foundation of China and Key Scientific Research Projects from the Universities of Henan Province.

Conflicts of Interest: The authors declare no conflict of interest.

\section{References}

1. Ruijter, E.; Orru, R.V.A. Multicomponent reactions-Opportunities for the pharmaceutical industry. Drug Discov. Today Technol. 2013, 10, e10-e15. [CrossRef] [PubMed]

2. Bora, U.; Saikia, A.; Boruah, R.C. A novel microwave-mediated one-pot synthesis of Indolizines via a three-component reaction. Org. Lett. 2003, 5, 435-438. [CrossRef] [PubMed]

3. Thompson, R.H. Naturally Occurring Quinones IV: Recents Advances; Champman \& Hall: London, UK, 1997. 
4. Benites, J.; Valderrama, J.A.; Bettega, K.; Pedrosa, R.C.; Calderon, P.B.; Verrax, J. Biological evaluation of donor-acceptor aminonaphthoquinones as antitumor agents. Eur. J. Med. Chem. 2010, 45, 6052-6057. [CrossRef] [PubMed]

5. Leyva, E.; López, L.; Cruz, R.; Espinosa-González, C.G. Synthesis and studies of the antifungal activity of 2-anilino-/2,3-dianilino-/2-phenoxy- and 2,3-diphenoxy-1,4-naphthoquinones. Res. Chem. Intermediat. 2016, 1-15. [CrossRef]

6. Jin, Y.R.; Ryu, C.K.; Moon, C.K.; Cho, M.R.; Yun, Y.P. Inhibitory effects of J78, a newly synthesized 1,4-naphthoquinone derivative, on experimental thrombosis and platelet aggregation. Pharmacology 2004, 70, 195-200. [CrossRef] [PubMed]

7. Sasaki, K.; Abe, H.; Yoshizaki, F. In vitro antifungal activity of naphthoquinone derivatives. Biol. Pharm. Bull. 2002, 25, 669-670. [CrossRef] [PubMed]

8. Shetgiri, N.P.; Kokitkar, S.V.; Sawant, S.N. Radermachera xylocarpa: The highły efficient source of lapachol and synthesis of its derivatives. Acta. Pol. Pharm. 2001, 58, 133-135. [PubMed]

9. Siripong, P.; Yahuafai, J.; Shimizu, K.; Ichikawa, K.; Yonezawa, S.; Asai, T.; Kanokmedakul, K.; Ruchirawat, S.; $\mathrm{Oku}, \mathrm{N}$. Antitumor activity of liposomal naphthoquinone esters isolated from thai medicinal plant: Rhinacanthus nasutus KURZ. Biol. Pharm. Bull. 2006, 29, 2279-2283. [CrossRef] [PubMed]

10. Han, L.; Huang, X.; Dahse, H.M.; Moellmann, U.; Fu, H.; Grabley, S.; Sattler, I.; Lin, W. Unusual naphthoquinone derivatives from the twigs of Avicennia marina. J. Nat. Prod. 2007, 70, 923-927. [CrossRef] [PubMed]

11. Bialonska, D.; Zjawiony, J.K. Aplysinopsins-marine indole alkaloids: Chemistry, bioactivity and ecological significance. Mar. Drugs 2009, 7, 66-183. [CrossRef] [PubMed]

12. Biswal, S.; Sahoo, U.; Sethy, S.; Kumar, H.K.S.; Banerjee, M. Indole: The molecule of diverse biological activities. Asian J. Pharm. Clin. Res. 2012, 5, 1-6.

13. Freidonk-Mueschenborn, E.; Fox, A. Resolution of concentration-response differences in onset of effect between subcutaneous and oral sumatriptan. Headach. J. Head Face Pain 2005, 45, 632-637. [CrossRef] [PubMed]

14. Generali, J.A.; Cada, D.J. Off-label drug uses-ondansetron: Postanesthetic shivering. Hosp. Pharm. 2009, 44, 670-671. [CrossRef]

Sample Availability: Samples of the compounds 4 are available from the authors.

(C) 2018 by the authors. Licensee MDPI, Basel, Switzerland. This article is an open access article distributed under the terms and conditions of the Creative Commons Attribution (CC BY) license (http://creativecommons.org/licenses/by/4.0/). 